\title{
Multivariate machine learning models for prediction of postoperative intestinal obstruction in patients underwent laparoscopic colorectal surgery: A retrospective observational study
}

\author{
Xueyan Li \\ Nanjing Medical University Affiliated Suzhou Hospital \\ Genshan Ma \\ Tumor Hospital Affiliated to Nantong University \\ Xiaobo Qian \\ Nanjing Medical University Affiliated Suzhou Hospital \\ Yamou Wu \\ Nanjing Medical University Affiliated Suzhou Hospital \\ Xiaochen Huang \\ Nanjing Medical University Affiliated Suzou Hospital \\ Chengyong Gu ( $\nabla$ gcygcy1979@163.com) \\ Nanjing Medical University Affiliated Suzhou Hospital
}

\section{Research article}

Keywords: machine learning, laparoscopic colorectal surgery, postoperative intestinal obstruction

Posted Date: December 12th, 2019

DOI: https://doi.org/10.21203/rs.2.18587/v1

License: (c) (i) This work is licensed under a Creative Commons Attribution 4.0 International License. Read Full License 


\section{Abstract}

Background

Machine learning may predict postoperative intestinal obstruction (POI) in patients underwent laparoscopic colorectal surgery for malignant lesions.

Methods

We used five machine learning algorithms (Logistic regression, Decision Tree, Forest, Gradient Boosting and gbm), analyzed by 28 explanatory variables, to predict POI. The total samples were randomly divided into training and testing groups, with a ratio of 8:2. The model was evaluated by the area operation characteristic curve (AUC), F1-Measure, accuracy, recall, and MSE under the receiver.

Results

A total of 637 patients were enrolled in this study, 122 (19.15\%) of them had POI. Gradient Boosting and gbm had the most accurate in training group and testing group respectively.The f1_score of Gradient Boosting was the highest in the training group ( $\mathrm{f}$ 1_score $=0.710526$ ), and the f1_score of gbm was the highest in the testing group (f1_score $=0.500000$ ). In addition, the results of the importance matrix of Gbdt algorithm model showed that the important variables that account for the weight of intestinal obstruction after the first five operations are time to pass flatus or passage of stool, cumulative dose of rescue opioids used in postoperative days 3 (POD 3), duration of surgery, height and weight.

Conclusions

Machine learning algorithms may predict the occurrence of POI in patients underwent laparoscopic colorectal surgery for malignant lesions, especially Gradient Boosting and GBM algorithms. Moreover, time to pass flatus or passage of stool, cumulative dose of rescue opioids used during POD 3, duration of surgery, height and weight play an important role in the development of POI.

\section{Background}

Postoperative intestinal obstruction (POI) is a symptomatic temporary impairment of gastrointestinal motility after abdominal surgery. Clinically, it may be characterized by bloating, pain, flatulence, fecal delay, nausea, and vomiting. A number of large randomized clinical trials showed that the incidence of intestinal obstruction after colorectal cancer surgery was about $2.28-5.48 \%$ $[1,2]$. POI may increase hospital stays and costs, which may result in serious complications [3]. For example, aspiration pneumonia may lead to $2.4-22 \%$ of early postoperative death [4]. Recently, laparoscopic methods have been shown to be associated with improved postoperative digestive recovery $[5,6]$.

However, part of patients underwent laparoscopic surgery still have a dynamic intestinal obstruction. In recent years, with the increase of environmental risk factors and the improvement of diagnostic techniques, the incidence of many diseases shows an ascending trend [7, 8]. "Machine learning" or "artificial intelligence" is the hotspot in predictive research. Shanmuga et al. used a deep learning method to analyze colorectal polyps in images, the results showed that tumor detection accuracy of the method using in colon images is up to $95 \%$ through the existing algorithm evaluation [9]. At the same time, Xu et al. applied the machine learning methods like support vector machine, which can effectively classify colon cancer patients with different prognosis [10]. In this study, we explored the application of machine learning methods to improve the prediction and classification of postoperative intestinal obstruction in patients underwent laparoscopic colorectal cancer surgery.

\section{Methods}

To investigate the predict effect of machine learning on POI underwent laparoscopic colorectal cancer surgery, we conducted a retrospective observational study at Nanjing Medical University Affiliated Suzhou Hospital.

\section{Participants}

We performed a retrospective analysis of consecutive patients aged 18 years or older who underwent laparoscopic colorectal surgery for malignant lesions from April 2016 to January 2017. Exclusion criteria were patients who underwent surgery other than 
laparoscopic colorectal surgery, converted to open surgery, robot-assisted laparoscopic colorectal surgery, and parenteral nutrition surgery. POI was defined as flatulence and/or fecal pass delay or oral intake intolerance on the third day after surgery and confirmed with radiographs that small and/or large intestinal dilatation on abdominal X-ray films.

\section{Anesthesia and operation management}

The surgeries were performed by six different surgeons, each with more than 200 experiences in laparoscopic colorectal surgery. Laparoscopic surgery includes single incision and conventional laparoscopic colorectal surgery. Anesthesia techniques were similar in all cases. There was no thoracic epidural analgesia. Intravenous midazolam凶sufentanil, propofol, and rocuronium were applied for induction of anesthesia, providing neuromuscular blockage for endotracheal intubation. Anesthesia was maintained with propofol, remifentanil, and sevoflurane. Opioids were routinely administered for postoperative pain 30 minutes prior to the end of surgery.

\section{Variables collection}

Data on patient demographics, social habits, comorbidities, intraoperative data (duration of surgery and anesthesia, type of surgery and anesthesia, quantity of intravenous infusion, estimated blood loss) and postoperative analgesia were collected (Maximum pain score [NRS] and cumulative dose of opioid used on the third day after surgery). All opioid administrations were converted to equivalent doses of intravenous morphine. The age-adjusted Charlson Comorbidity Index was used to assess comorbidity. The original Charlson Comorbidity Index was coined in 1987 and it was calculated by summing the weighted scores of 19 medical conditions. Since age was determined to be an important factor in overall survival, the patient's age subsequently acted as a correction variable in the final Charlson index score. It is reported that this modification of the Charlson Comorbidity Index called age adjustment has better predictive effect on hospital mortality and adverse events than other versions of the Charlson Comorbidity Index. Events such as postoperative wound dehiscence were also recorded.

\section{Machine learning algorithm}

Logistic regression is one of the most commonly used and most classical classification methods in machine learning. Although it is called a regression model, it deals with classification problems. This is mainly because its essence is a linear model plus a mapping function sigmoid, which maps the continuous results obtained by the linear model to discrete models.

Decision tree learning is a method of approaching the objective function of discrete value in which the learned function is represented as a decision tree. A decision tree classifies an instance by arranging instances from a root node to a leaf node. The leaf node is the class to which the instance belongs. Each node on the tree specifies a test for an attribute of the instance, and each subsequent branch of the node corresponds to a possible value for the attribute. The classification to an instance starts from the root of the tree, testing the properties of the node, and then move down the branches corresponding to the property values of the given instance. This process is then repeated on the subtree of the new root node.

Random forest, as the name implies, establishes a forest randomly. There are many decision trees in the forest, and there is no correlation between each decision tree in the random forest. After the forest is gotten, when a new input sample enters, each decision tree in the forest makes a separate judgment to classify the sample (for the classification algorithm), and the sample is predicted to be the classification that has been chosen for the most times.

Gbdt is an iterative decision tree algorithm consisting of multiple decision trees. The conclusions of all trees are added together to make the final answer.

Lightgbm ( $(\mathrm{gbm})$ is another implementation method of Gbdt, which adopts two new strategies based on Gbdt. Gradient-based OneSide Sampling (GOSS) is that, although Gbdt has no data weight, each data instance has different gradients. According to the definition of computing information gain, the instance with larger gradient has greater influence on the information gain. Thus, samples with large gradients should be kept (pre-set thresholds or highest percentiles) and samples with small gradients should be randomly removed during downsampling whenever possible. Exclusive Feature Bundling (EFB) means that many features are almost mutually exclusive especially in sparse feature spaces, and we can bundle mutually exclusive features. Finally, we reduce the bundling problem to the graph coloring problem and obtain an approximate solution through the greedy algorithm. 
Python programming language (Python Software Foundation, version 3.6) were used for our analysis. The following packages for machine learning were used: Scikit-learn (https://github.com/scikit-learn/scikit-learn) and lightgbm

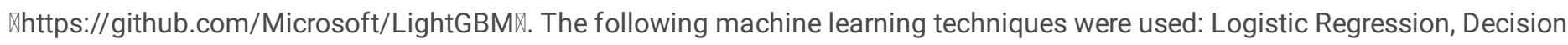
Tree, Gradient Boosting, and Lightgbm. 31 explanatory variables were applied for machine learning analysis. Our samples were randomly divided into training and test groups at a ratio of 8:2. Machine learning techniques were evaluated and the prediction accuracy was compared with the following methods, we calculated the area under the receiver operator characteristic curves (AUC) and compared the F1-Measure, accuracy, recall rate, and MSE. The missed values of the variables were estimated with multiple imputations. Values were normalized and scaled to 0-1.

F1-Measure evaluation indicator is often used in information retrieval and natural language processing. It is a comprehensive evaluation index based on Precision and Recall $(F 1=2 r p /(r+p))$, which is defined as follows: Where $r$ is recall and $p$ is precision. Accuracy rate indicates the proportion of the correctly classified samples in the total samples. Precision rate indicates the probability of the correctly predicted positive samples among all the samples predicted as positive samples. Recall rate indicates the probability of correctly predicted positive samples among all the original positive samples. The full name of the ROC is the " receiver operating characteristic curve ". AUC is defined as the area under the ROC curve.

\section{Results}

A total of 637 patients were included in the study, and 122(19.15\%) cases had POI. The average age of patients with POI was $63.7 \pm$ 12.5 years old, and the average age of patients without POI was $61.4 \pm 11.6$ years old. $62(50.8 \%)$ of POI patients were male and 60 $(49.2 \%)$ were female. (See in Table 1 ) 
Table 1

Characteristics of patients

\begin{tabular}{|c|c|c|c|}
\hline POSTOPERATIVE.ILEUS & NO & YES & P-value \\
\hline $\mathrm{n}$ & 515 & 122 & \\
\hline AGE(years) & $61.4 \pm 11.6$ & $63.7 \pm 12.5$ & 0.027 \\
\hline WEIGHT(kg) & $62.6 \pm 10.9$ & $60.6 \pm 11.7$ & 0.052 \\
\hline $\mathrm{HEIGHT}(\mathrm{cm})$ & $162.5 \pm 8.8$ & $160.9 \pm 9.9$ & 0.077 \\
\hline BMII $\left(\mathrm{kg} / \mathrm{m}^{2}\right)$ & $23.7 \pm 3.3$ & $23.3 \pm 3.3$ & 0.088 \\
\hline SURGERY.TIME(min) & $140.1 \pm 49.0$ & $160.2 \pm 75.5$ & 0.001 \\
\hline ANESTHESIA.TIME(min) & $185.5 \pm 51.3$ & $205.8 \pm 74.4$ & $<0.001$ \\
\hline LOWEST.INTRAOPERATIVE.CORE.TEMPERATURE $\left({ }^{\circ} \mathrm{C}\right)$ & $35.5 \pm 0.5$ & $35.3 \pm 0.5$ & 0.004 \\
\hline INTRAVENOUS.FLUIDS(mL) & $1058.5 \pm 397.5$ & $1181.7 \pm 483.3$ & 0.002 \\
\hline TYMPANIC.TEMPERATURE.ON.PACU.ICU.ADMISSION $\left({ }^{\circ} \mathrm{C}\right)$ & $35.7 \pm 0.6$ & $35.6 \pm 0.6$ & $<0.001$ \\
\hline DOSE.OF.OPIOIDS(morphine equivalents [mg]) & $16.3 \pm 14.2$ & $24.1 \pm 19.2$ & $<0.001$ \\
\hline AGE.ADJUSTED.CHARLSON.COMORBIDITY.INDEX & $4.1 \pm 1.5$ & $4.7 \pm 1.8$ & 0.003 \\
\hline MAXIMUM.PAIN.NRS.SCORE.DURING.POD3 & $5.6 \pm 1.8$ & $6.0 \pm 1.7$ & 0.014 \\
\hline TIME.TO.PASS.FLATUS.OR.PASSAGE.OF.STOOL(Day) & $2.8 \pm 0.9$ & $3.9 \pm 2.0$ & $<0.001$ \\
\hline TIME.TO.THE.FIRST.SOLID.FOOD.INTAKE(Day) & $2.7 \pm 1.1$ & $3.6 \pm 2.7$ & $<0.001$ \\
\hline SEX & & & 0.570 \\
\hline female & $247(48.0 \%)$ & $62(50.8 \%)$ & \\
\hline male & $268(52.0 \%)$ & $60(49.2 \%)$ & \\
\hline ASA.CLASSIFICATION & & & $<0.001$ \\
\hline 0 & $125(24.3 \%)$ & $9(7.4 \%)$ & \\
\hline 1 & $264(51.3 \%)$ & $79(64.8 \%)$ & \\
\hline 2 & $120(23.3 \%)$ & $31(25.4 \%)$ & \\
\hline 3 & $6(1.2 \%)$ & $3(2.5 \%)$ & \\
\hline ALCOHOL.ABUSE & & & 0.635 \\
\hline No & $408(79.2 \%)$ & $99(81.1 \%)$ & \\
\hline Yes & $107(20.8 \%)$ & $23(18.9 \%)$ & \\
\hline SMOKING.HISTORY & & & 0.196 \\
\hline No & 427 (82.9\%) & $107(87.7 \%)$ & \\
\hline Yes & $88(17.1 \%)$ & $15(12.3 \%)$ & \\
\hline TYPE.OF.SURGERY & & & 0.242 \\
\hline left-sided colectomy & $147(28.5 \%)$ & $35(28.7 \%)$ & \\
\hline right-sided colectomy & $197(38.3 \%)$ & $36(29.5 \%)$ & \\
\hline
\end{tabular}




\begin{tabular}{|c|c|c|c|}
\hline POSTOPERATIVE.ILEUS & NO & YES & P-value \\
\hline rectal surgery & $162(31.5 \%)$ & $48(39.3 \%)$ & \\
\hline others & $9(1.7 \%)$ & $3(2.5 \%)$ & \\
\hline PRESENCE.OF.STOMY & & & 0.180 \\
\hline No & $480(93.2 \%)$ & $109(89.3 \%)$ & \\
\hline Yes & $35(6.8 \%)$ & $13(10.7 \%)$ & \\
\hline OPERATOR & & & 0.093 \\
\hline 0 & $204(39.6 \%)$ & $37(30.3 \%)$ & \\
\hline 1 & $74(14.4 \%)$ & $22(18.0 \%)$ & \\
\hline 2 & $135(26.2 \%)$ & $26(21.3 \%)$ & \\
\hline 3 & $61(11.8 \%)$ & $22(18.0 \%)$ & \\
\hline 4 & $33(6.4 \%)$ & $13(10.7 \%)$ & \\
\hline 5 & $8(1.6 \%)$ & $2(1.6 \%)$ & \\
\hline TYPE.OF.ANESTHESIA & & & 1.000 \\
\hline SEVO or DES only & $444(86.2 \%)$ & $105(86.1 \%)$ & \\
\hline TIVA/ & $30(5.8 \%)$ & $7(5.7 \%)$ & \\
\hline SEVO + REMI & $41(8.0 \%)$ & $10(8.2 \%)$ & \\
\hline INTRAOPERATIVE.HYPOTHERMIA & & & 0.044 \\
\hline No & $94(18.3 \%)$ & $13(10.7 \%)$ & \\
\hline Yes & $421(81.7 \%)$ & $109(89.3 \%)$ & \\
\hline ADMISSION.TO.ICU & & & 0.256 \\
\hline No & $469(91.1 \%)$ & $107(87.7 \%)$ & \\
\hline Yes & $46(8.9 \%)$ & $15(12.3 \%)$ & \\
\hline HYPOTHERMIA.ON.PACU.ICU.ADMISSION & & & 0.040 \\
\hline No & $208(40.4 \%)$ & $37(30.3 \%)$ & \\
\hline Yes & $307(59.6 \%)$ & $85(69.7 \%)$ & \\
\hline IVPCA.REFILL & & & 0.133 \\
\hline No & $511(99.2 \%)$ & $119(97.5 \%)$ & \\
\hline Yes & $4(0.8 \%)$ & $3(2.5 \%)$ & \\
\hline WOUND.DEHISCENCE & & & 0.028 \\
\hline No & $512(99.4 \%)$ & $118(96.7 \%)$ & \\
\hline Yes & $3(0.6 \%)$ & $4(3.3 \%)$ & \\
\hline \multicolumn{4}{|c|}{$\begin{array}{l}\text { BMI: body mass index; SEV: sevoflurane; DES: desflurane; TIVA: total intravenous anesthesia; REMI: remifentanil; PACU: } \\
\text { postanesthesia care unit; ICU:intensive care unit; NRS: numerical rating scale; POD: postoperative days. Values are means } \pm \\
\text { standard deviation, medians (interquartile range), or numbers }\end{array}$} \\
\hline
\end{tabular}

The importance matrix of the Gbdt algorithm model is shown in Fig. 1. The top five important variables that account for the weight of postoperative intestinal infarction included time to pass flatus or passage of stool, cumulative dose of rescue opioids used during POD 3, duration of surgery and height and weight. In the correlation heat map (Fig. 2), we found that height and weight were weakly 
negatively correlated with intestinal infarction. time to pass flatus or passage of stool, cumulative dose of rescue opioids used during POD 3 and duration of surgery were weakly positively correlated with intestinal infarction, and positive correlation index of time to pass flatus or passage of stool was the highest, that was 0.33 .

The comparation of models constructed by the five machine learning algorithms in the training set were shown in Table 1 and Fig. 1 . The logistic regression model showed that AUC value $=0.8177$, accuracy $=0.8468$, precision $=0.7317$, recall $=0.3093, f 1 \_s c o r e=$ 0.4348 and $\mathrm{MSE}=0.1532$; the decision tree model shows that $\mathrm{AUC}$ value $=0.7820$, accuracy $=0.8507$, precision $=0.8000$, recall $=$ $0.2887, f 1 \_s c o r e=0.4242$ and MSE $=0.1493$, the random forest model shows that $A U C$ value $=0.9660$, accuracy $=0.8802$, precision $=$ 0.9737 , recall $=0.3814, \mathrm{f} 1 \_$score $=0.5481$ and MSE $=0.1198$, the GradientBoosting model shows that $\mathrm{AUC}$ value $=0.9650$, accuracy $=$ 0.9136 , precision $=0.9818$, recall $=0.5567, f 1 \_$score $=0.7105$ and MSE $=0.1238$; the gbm model shows that AUC value $=0.9446$, accuracy $=0.8860$, precision $=0.9149$, recall $=0.4433, f 1 \_$score $=0.5972$ and MSE $=0.1611$. (See in Table 2 and Fig. 3)

Table 2

Forecast results of the training group

\begin{tabular}{|lllllll|}
\hline & accuracy & precision & recall & f1_score & AUC & MSE \\
\hline Logistic & 0.8468 & 0.7317 & 0.3092 & 0.4349 & 0.8177 & 0.1532 \\
\hline DecisionTree & 0.8507 & 0.8000 & 0.2887 & 0.4242 & 0.7820 & 0.1493 \\
\hline Forest & 0.8802 & 0.9737 & 0.3814 & 0.5481 & 0.9660 & 0.1198 \\
\hline GradientBoosti & 0.9136 & 0.9818 & 0.5567 & 0.7105 & 0.9650 & 0.1238 \\
\hline Gbm & 0.8861 & 0.9149 & 0.4433 & 0.5972 & 0.9446 & 0.1611 \\
\hline
\end{tabular}

The comparation of models constructed by the five machine learning algorithms in the test set were shown in Table 1 and Fig. 1. The logistic regression model showed that AUC value $=0.7445$, accuracy $=0.8125$ precision $=0.5556$, recall $=0.2000, f 1 \_s c o r e=0.2941$ and MSE $=0.1875$; the decision tree model showed that $\mathrm{AUC}$ value $=0.7748$, accuracy $=0.7969$, precision $=0.4615$, recall $=$ $0.2400, f 1$ fscore $=0.3158$ and MSE $=0.2031$, the random forest model showed that AUC value $=0.7348$, accuracy $=0.8203$, precision $=0.6667$, recall $=0.1600, f 1$ score $=0.2581$ and MSE $=0.1797$, the GradientBoosting model showed that AUC value $=0.7631$, accuracy $=0.8359$, precision $=0.6667$, recall $=0.3200, f 1$ score $=0.4324$ and MSE $=0.1641$, the gbm model showed that AUC value $=0.7550$, accuracy $=0.8594$, precision $=0.8182$, recall $=0.3600, f 1 \_$score $=0.5000$ and MSE $=0.1406$. (See in Table 3 and Fig. 4)

Table 3

Forecast results of the testing group

\begin{tabular}{|lllllll|}
\hline & accuracy & precision & recall & f1_score & AUC & MSE \\
\hline Logistic & 0.8125 & 0.5556 & 0.2000 & 0.2941 & 0.7445 & 0.1875 \\
\hline DecisionTree & 0.7969 & 0.4615 & 0.2400 & 0.3158 & 0.7748 & 0.2031 \\
\hline forest & 0.8203 & 0.6667 & 0.1600 & 0.2581 & 0.7348 & 0.1797 \\
\hline GradientBoosting & 0.8359 & 0.6667 & 0.3200 & 0.4324 & 0.7631 & 0.1641 \\
\hline gbm & 0.8594 & 0.8182 & 0.3600 & 0.5000 & 0.7550 & 0.1406 \\
\hline
\end{tabular}

In this study, the final parameters of GradientBoosting were penalty='I2',tol $=0.000001, C=0.1$, fit_intercept $=$ True, intercept_scaling = 1 ,class_weight $=$ None,max_iter $=100$, multi_class='ovr',verbose $=0$,warm_start $=$ False,n_jobs $=1$, the final parameters of DecisionTree were ssplitter='best', max_depth $=3$, min_samples_split $=30$, min_samples_leaf $=2$, min_weight_fraction_leaf $=0.01$. The rest were shown in Table 4. 
Table 4

Functions, Packages, and Tuning Parameters in the anaconda Software Used for Each Machine Learning Algorithm

\begin{tabular}{|c|c|c|c|}
\hline Algorithm & Classifier & Package & Tuning Parameters \\
\hline $\begin{array}{l}\text { Logistic } \\
\text { regression }\end{array}$ & LogisticRegression & $\begin{array}{l}\text { Sklearn } \\
0.19 .1\end{array}$ & $\begin{array}{l}\text { penalty='I2',tol }=0.000001, C=0.1, \text { fit_intercept }= \\
\text { True,intercept_scaling }=1, \text { class_weight }=\text { None,max_iter }= \\
100, \text { multi_class='ovr',verbose }=0, \text { warm_start }=\text { False, } n \_j o b s=1\end{array}$ \\
\hline DecisionTree & DecisionTreeClassifier & $\begin{array}{l}\text { Sklearn } \\
0.19 .1\end{array}$ & $\begin{array}{l}\text { splitter='best',max_depth }=3 \text {,min_samples_split }= \\
30 \text {,min_samples_leaf }=2, \text { min_weight_fraction_leaf }=0.01\end{array}$ \\
\hline forest & RandomForestClassifier & $\begin{array}{l}\text { Sklearn } \\
0.19 .1\end{array}$ & $\begin{array}{l}\text { n_estimators }=50, \text { n_jobs }=-1, \text { min_samples_split }= \\
20, \text { min_samples_leaf }=2 \text {,random_state }=41\end{array}$ \\
\hline GradientBoosting & GradientBoostingClassifier & $\begin{array}{l}\text { Sklearn } \\
0.19 .1\end{array}$ & $\begin{array}{l}\text { learning_rate }=0.1, \text { n_estimators }=20 \text {,max_depth }=3 \text {, } \\
\text { min_samples_split }=30 \text {,min_samples_leaf }=5\end{array}$ \\
\hline $\mathrm{gbm}$ & Igb.LGBMClassifier & $\begin{array}{l}\text { lightgbm } \\
2.2 .0\end{array}$ & $\begin{array}{l}\text { boosting_type='gbdt',objective='binary', metrics='auc',learning_rate } \\
=0.1, \text { n_estimators }=30 \text {, max_depth }=2 \text {, bagging_fraction = } \\
0.8 \text {,feature_fraction }=0.2\end{array}$ \\
\hline
\end{tabular}

\section{Discussion}

Although the promotion of laparoscopic technique has significantly reduced the incidence of dynamic intestinal obstruction, postoperative dynamic intestinal obstruction still occurs after colorectal cancer operations. Kronberg et al. studied patients underwent laparoscopic surgery and reported a $10.2 \%$ incidence of postoperative dynamic intestinal obstruction [11]. In this study, we used five machine learning methods to predict postoperative intestinal obstruction in patients undergoing laparoscopic colorectal cancer surgery. The results showed that AUC, accuracy rate and precision rate of GradientBoosting and gbm algorithms were the highest in the training and test groups compared to the other two algorithms. The MSE of forest was the lowest (0.1198) in the training group. The f1_score in GradientBoosting was the highest in the training group, and it was 0.7105 . The f1_score of $X$ algorithm in gbm was the highest in the test group, which was 0.5. In the test group, gbm has the lowest MSE value and the highest accuracy. In addition, the importance matrix of the Gbdt algorithm model showed that the top five important variables that account for the weight of postoperative intestinal infarction were time to pass flatus or passage of stool, cumulative dose of rescue opioids used during POD 3, duration of surgery, height and weight. In the correlation heat map, we found that height and weight were weakly negatively correlated with intestinal infarction, and time to pass flatus or passage of stool, cumulative dose of rescue opioids used during POD 3, duration of surgery were weakly positively correlated with intestinal infarction and positive correlation index of time to pass flatus or passage of stool was the highest, which was 0.33 .

Logistic regression is a commonly used machine learning method used to estimate the possibility of a certain objective. It has little calculation amount, short calculation time, and takes low storage resources during classification. However, when the characteristic space is large, the performance of logistic regression is not well. Since that only 28 feature variables were included in this study, the prediction model can be constructed well.(Training: $A U C=0.8177$; and test $A U C=0.7445$ )

The decision tree learning algorithm is a process of recursive selection of the classification for each sub-data set. In this study, we used the classification and regression tree (CART) algorithm in the decision tree model. However, this algorithm can easily generate complex tree structures in the training data, resulting in over-fitting. Since the results of the decision tree algorithm in study are stable, there was no overfitting. (Training: $A U C=0.7820$; and test $A U C=0.7748$ )

The random forest has many advantages: 1 . Each tree selects part of the samples and features, thus over-fitting can be avoided to a certain extent. 2. Each tree randomly selects samples and features, so it has good anti-noise ability and stable performance. 3. It can handle high dimensional data and does not need to make feature selection. 4. Random forest is suitable for parallel processing, and the implementation is relatively simple. However, its parameters are complex and the model training and prediction are slow. Random algorithms in this study may cause over-fitting. (Training: $A U C=0.9660$; and test AUC $=0.7348$ )

GBDT integrates several models with common performance (usually deeply fixed decision tree) into a model with better performance. It has the natural processing ability for mixed data and has strong predictive ability. LightGBM is a tree-based gradient boosting 
framework that supports efficient parallel training. The results of this study also showed that the GradientBoosting and gbm performed better than the other two algorithms.

$\mathrm{POI}$ is still a common complication after colorectal surgery and its pathophysiology remains unclear [12]. A cohort study including nearly 28,000 patients reported a POI incidence of $12.7 \%$ after colon surgery. The incidence of postoperative intestinal obstruction was higher in obese patients [13]. In a small series of reports, according to Parker et al postoperative intestinal obstruction patients are basically obese [14]. The reason may be that the intestines of obese patients contain more mesenteric fat, which makes it more difficult to operate, and may cause damage to the intestine during surgery. This is consistent with our findings.

General anesthesia, especially the application of opioids, has been proved to be able to affect bowel movements [15]. Opioids such as morphine can bind to the gastrointestinal $\mu$ receptor and inhibit gastrointestinal motility. The study of Boelens et al has shown that the incidence of $\mathrm{POI}$ in patients administered with opioids after colorectal cancer surgery was significantly higher than that of patients who didn't use opioids after operation [16]. In recent years, ideal therapeutic effect had been obtained by pre-operatively administering to the patient with $\mu$ receptor agonist alvimopan for the prevention and treatment of POI [17]. In addition, Pillai et al. conducted a randomized controlled study of perioperative fluid management and esophageal ultrasound Doppler detection, and the results showed that optimizing perioperative fluid intake was correlated with enhancing rapidly restored intestinal function and reducing the incidence of other complications [18]. Our results also suggested that the application of opioids and fluid infusion may play an important role in the occurrence and development of POI.

In addition, a recent retrospective study involving 11,397 patients who underwent open or laparoscopic colectomy showed that the high age-adjusted Charlson comorbidity index score was an independent predictor of POI prolongation [19]. Long operating time was a risk factor for $\mathrm{POI}$ in colorectal surgery [20]. Long operative time may indicate prolongation, technical difficulties, and/or increased inflammatory response, any of which can directly induce the occurrence of POI [21,22]. This is similar to our results.

This study has several potential limitations. A major limitation is the use of retrospective methods for data collection. Moreover, in addition to the dose of opioid used in the first 3 days after surgery, there may be doses at other times that contribute to the POI. Moreover, although we can quantify the weight of each variable on the postoperative intestinal obstruction through machine learning methods, there are many variables that cannot be intervened. We can prevent and pay attention to these variables according to their risk of causing postoperative intestinal obstruction. In addition, this study performed only internal verification and no external verification. Therefore, we need to collect more perioperative data based on etiology and explore a more efficient predictive model in further study.

\section{Conclusions}

Machine learning algorithms can effectively predict postoperative intestinal obstruction in patients who underwent laparoscopic colorectal cancer surgery. Moreover, time to pass flatus or passage of stool, cumulative dose of rescue opioids used during POD 3, duration of surgery, height and weight are the top five variables that account for important weight of POI.

\section{List Of Abbreviations}

POI: ostoperative intestinal obstruction

AUC: area operation characteristic curve

GOSS: Gradient-based One-Side Sampling

EFB: Exclusive Feature Bundling

BMI: body mass index

SEV: sevoflurane

DES: desflurane

TIVA: total intravenous anesthesia 
REMI: remifentanil

PACU: postanesthesia care unit

ICU: intensive care unit

NRS: numerical rating scale

POD: postoperative days

CART: assification and regression tree.

\section{Declarations}

\section{Ethics approval and consent to participate}

Ethical approval for this study was waived by Suzhou Municipal Hospital Institutional Review Board , considering the retrospective design.

\section{Consent for publication}

Not applicable.

\section{Availability of data and materials}

The datasets used and/or analysed during the current study are available from the corresponding author on reasonable request.

\section{Competing interests}

The authors declare that they have no competing interests.

\section{Funding}

This study was funded by the Science and technology development fund of Nanjing Medical University. The funding agent plays no role in the design of the study and collection, analysis, and interpretation of data and in writing the manuscript.

\section{Authors' contributions}

CG conceived and designed the study. XL, GM, XQ, YW, and XH performed this study and analyzed the data. XL and GM wrote the manuscript. CG reviewed and edited the manuscript. All authors read and approved the final manuscript.

\section{Acknowledgements}

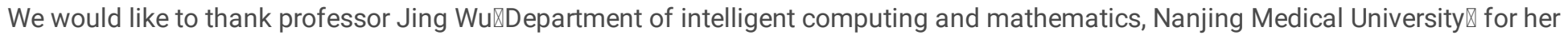
extensive assistance with the study. Furthermore, We would like to thank the general surgeons of Suzhou Municipal Hospital (North District) for their support.

\section{References}

1. Braga M, Vignali A, Gianotti L, Zuliani W, Radaelli G, Gruarin P, et al. Laparoscopic versus open colorectal surgery: a randomized trial on short-term outcome. Ann Surg. 2002;236:759-66.

2. Braga M, Vignali A, Zuliani W, Frasson M, Di Serio C, Di Carlo V. Laparoscopic versus open colorectal surgery: cost-benefit analysis in a single-center randomized trial. Ann Surg. 2005;242:890-5.

3. Iyer S, Saunders WB, Stemkowski S. Economic burden of postoperative ileus associated with colectomy in the United States. J Manag Care Pharm. 2009;15:485-94. 
4. Ellozy SH, Harris MT, Bauer JJ, Gorfine SR, Kreel I. Early postoperative small-bowel obstruction: a prospective evaluation in 242 consecutive abdominal operations. Dis Colon Rectum. 2002;45:1214-7.

5. van der Pas MH, Haglind E, Cuesta MA, Fürst A, Lacy AM, Hop WC, et al. Laparoscopic versus open surgery for rectal cancer (COLOR II): short-term outcomes of a randomised, phase 3 trial. Lancet Oncol. 2013;14:210-8.

6. Shussman N, Brown MR, Johnson MC, Da Silva G, Wexner SD, Weiss EG. Does nasogastric tube decompression get used less often with laparoscopic and hand-assisted compared with open colectomy? Surg Endosc. 2013;27:4564-8.

7. Shameer K, Johnson KW, Glicksberg BS, Dudley JT, Sengupta PP. Machine learning in cardiovascular medicine: are we there yet? Heart. 2018;104:1156-64.

8. Bibault J-E, Giraud P, Burgun A. Big Data and machine learning in radiation oncology: State of the art and future prospects. Cancer Lett. 2016;382:110-7.

9. Shanmuga Sundaram P, Santhiyakumari N. An Enhancement of Computer Aided Approach for Colon Cancer Detection in WCE Images Using ROI Based Color Histogram and SVM2. J Med Syst. 2019;43:29.

10. Xu G, Zhang M, Zhu H, Xu J. A 15-gene signature for prediction of colon cancer recurrence and prognosis based on SVM. Gene. 2017;604:33-40.

11. Kronberg U, Kiran RP, Soliman MSM, Hammel JP, Galway U, Coffey JC, et al. A characterization of factors determining postoperative ileus after laparoscopic colectomy enables the generation of a novel predictive score. Ann Surg. 2011;253:78-81.

12. Holte K, Kehlet H. Postoperative ileus: a preventable event. Br J Surg. 2000;87:1480-93.

13. Moghadamyeghaneh Z, Hwang GS, Hanna MH, Phelan M, Carmichael JC, Mills S, et al. Risk factors for prolonged ileus following colon surgery. Surg Endosc. 2016;30:603-9.

14. Parker AS, Lewis R, Heckman MG, Diehl NH, Brisson T, Pak R, et al. Evaluation of the impact of body mass index on outcome among renal mass patients treated with hand-assisted laparoscopic radical nephrectomy. J Endourol. 2008;22:301-6.

15. Cali RL, Meade PG, Swanson MS, Freeman C. Effect of Morphine and incision length on bowel function after colectomy. Dis Colon Rectum. 2000;43:163-8.

16. Boelens PG, Heesakkers FFBM, Luyer MDP, van Barneveld KWY, de Hingh IHJT, Nieuwenhuijzen GAP, et al. Reduction of postoperative ileus by early enteral nutrition in patients undergoing major rectal surgery: prospective, randomized, controlled trial. Ann Surg. 2014;259:649-55.

17. Zhu P, Liang Z, Fu J, Chen W, Wang Z, Jiang H, et al. Procalcitonin in abdominal exudate to predict prolonged postoperative ileus following colorectal carcinoma surgery. Int J Biol Markers. 2013;28:187-91.

18. Pillai P, McEleavy I, Gaughan M, Snowden C, Nesbitt I, Durkan G, et al. A double-blind randomized controlled clinical trial to assess the effect of Doppler optimized intraoperative fluid management on outcome following radical cystectomy. J Urol. 2011;186:2201-6.

19. Tian Y, Xu B, Yu G, Li Y, Liu H. Age-adjusted charlson comorbidity index score as predictor of prolonged postoperative ileus in patients with colorectal cancer who underwent surgical resection. Oncotarget. 2017;8:20794-801.

20. Scheer A, Martel G, Moloo H, Sabri E, Poulin EC, Mamazza J, et al. Laparoscopic colon surgery: does operative time matter? Dis Colon Rectum. 2009;52:1746-52.

21. Mattei P, Rombeau JL. Review of the pathophysiology and management of postoperative ileus. World J Surg. 2006;30:1382-91.

22. Vather R, O'Grady G, Bissett IP, Dinning PG. Postoperative ileus: mechanisms and future directions for research. Clin Exp Pharmacol Physiol. 2014;41:358-70.

\section{Figures}




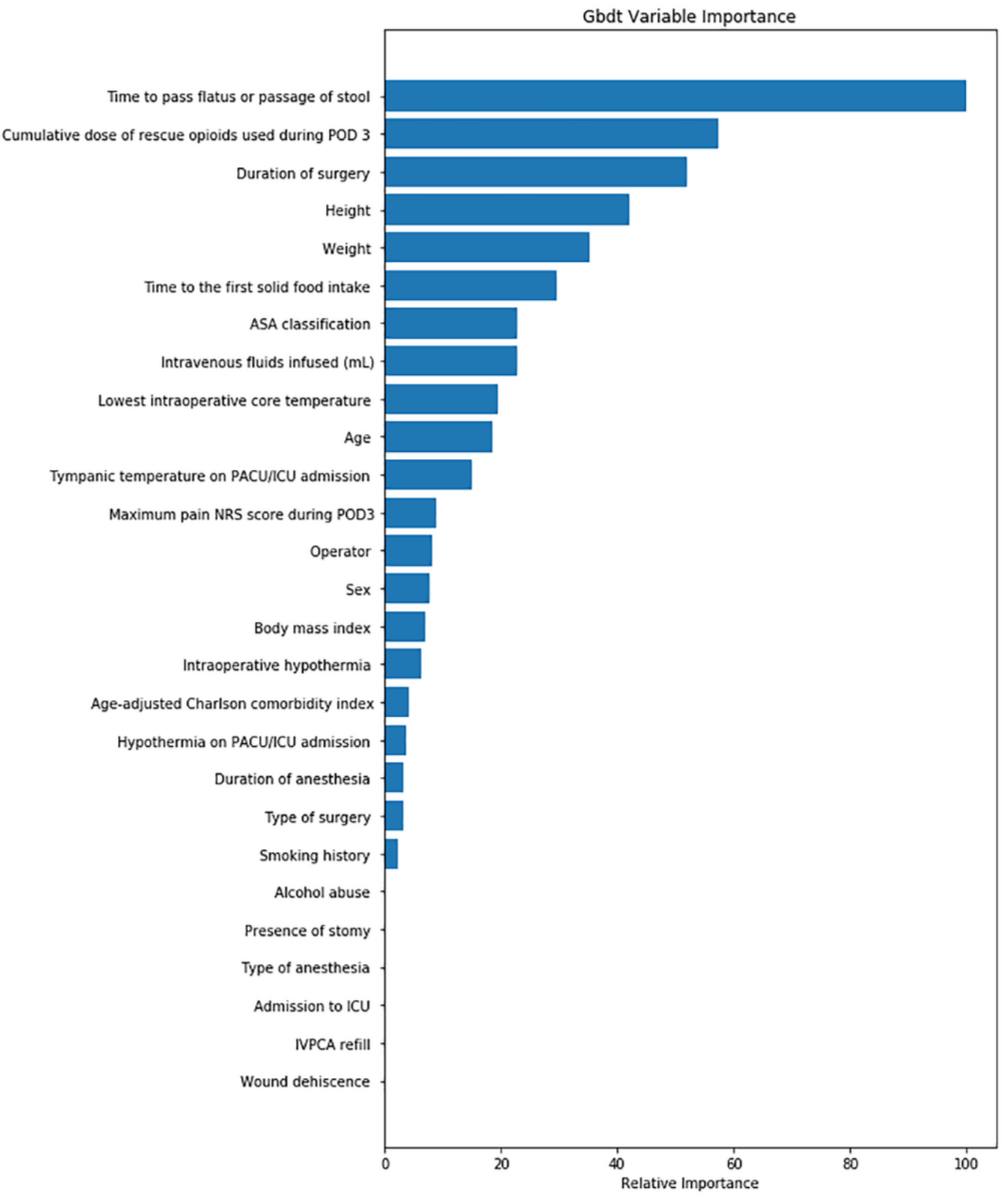

\section{Figure 1}

Variable importance of features included in machine learning algorithm for prediction of postoperative ileus 


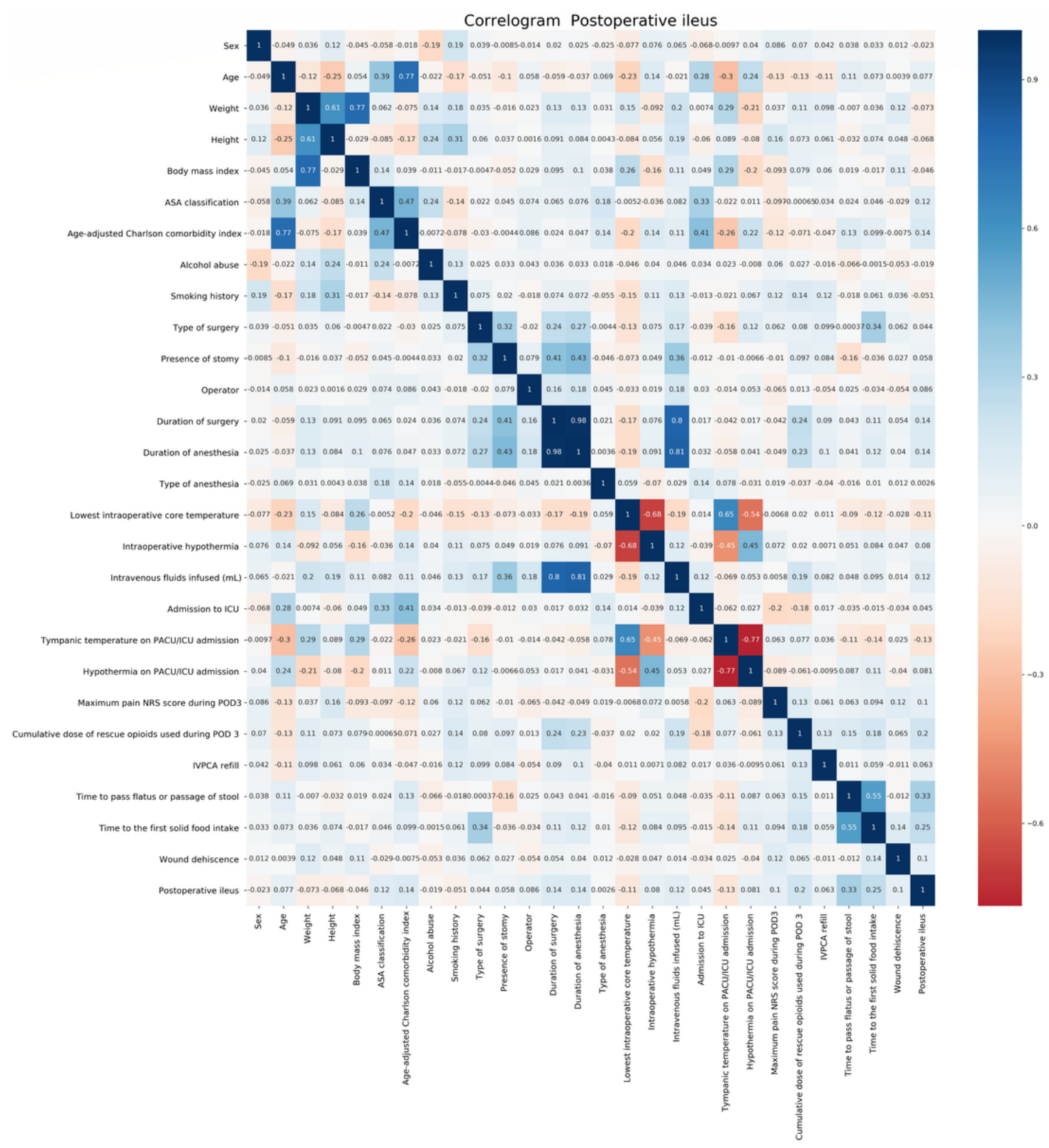

Figure 2

Correlation analysis of various factors 

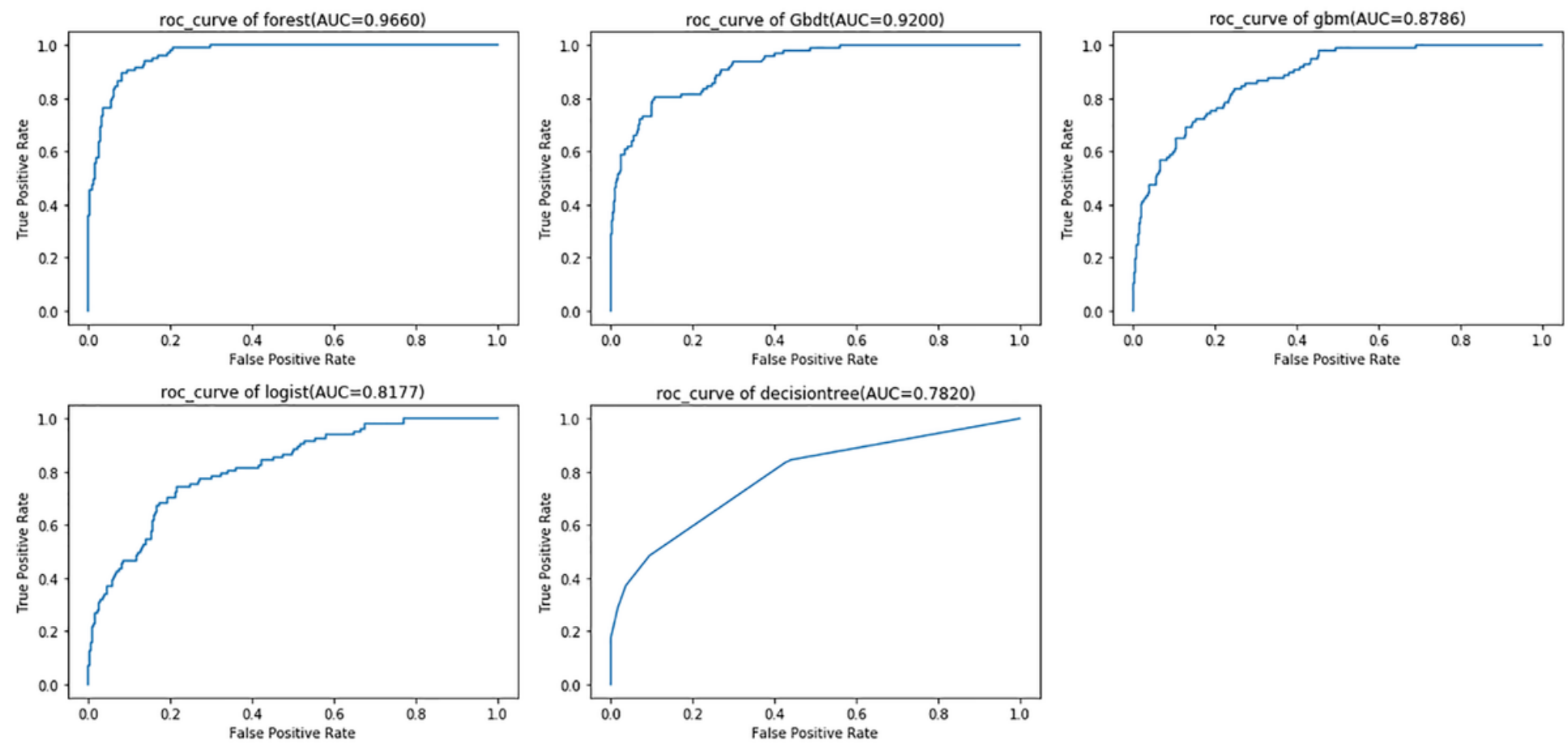

\section{Figure 3}

Machine learning algorithm for prediction of postoperative ileus in the training group
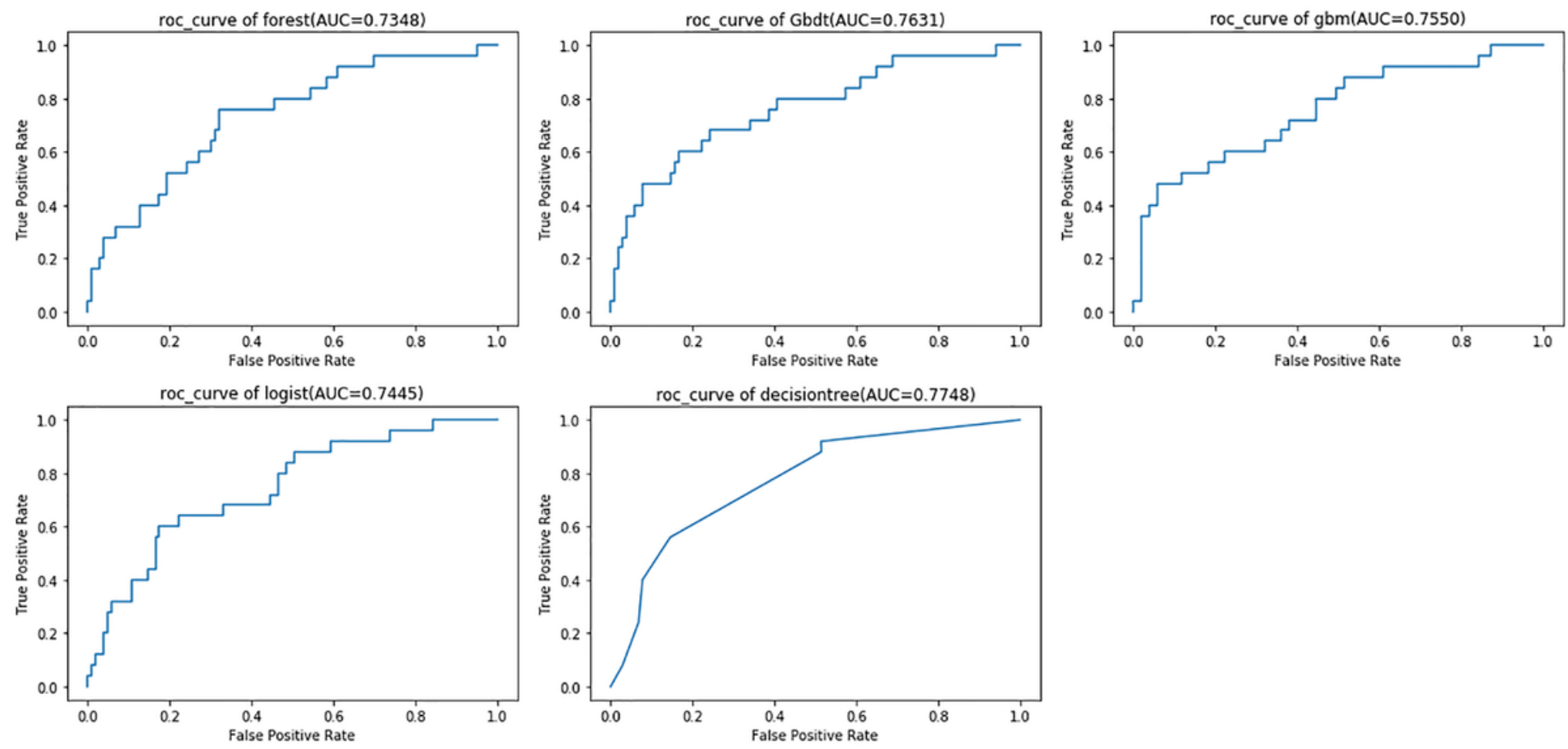

\section{Figure 4}

Machine learning algorithm for prediction of postoperative ileus in the testing group 